\title{
Rabies Pre-Exposure Vaccination in Rabies Endemic Countries
}

\section{Claudius Malerczyk*}

Medical Affairs Middle East \& Africa, Novartis Vaccines and Diagnostics, Marburg, Germany

Rabies is a viral zoonosis that causes an acute encephalitis not only in a variety of animal species but also in humans. It is endemic worldwide, but human death toll greatly differs from continent to continent. The World Health Organization estimates the number of human death cases to be in the range of about 30-60,000 per year [1]. While in Europe and North America the number of human cases is low, it is highest in Asia and Africa, where dograbies is highly endemic. In Asia, an estimated 30,000 deaths occur annually. The highest burden of disease is probably seen in India, where up to 20,000 people die of rabies every year. Children in endemic areas are at particular high risk. About 40 percent of all animal bite exposures are seen in children below the age of 15 [2]. In Thailand up to $30 \%$ of all children will have been bitten by a dog by the age of 15 [3]. Rabies is virtually always fatal once clinical symptoms have developed; a few single cases of survival have been documented, but a break-through has still not occurred. Nevertheless, rabies is preventable by vaccination, either before or after an exposure has occurred. Post-exposure prophylaxis (PEP) has been shown highly efficacious when administered in time after exposure and appropriately. This includes proper wound cleaning, administration of vaccine and - according to the category of exposure - administration of rabies immunoglobulin (RIG). An often forgotten alternative to PEP is pre-exposure prophylaxis (PrEP), which consists of three doses of vaccine given on days 0,7 , and 21 or 28 . A person vaccinated with a full PrEP schedule can be regarded as protected for several years, and in case of an exposure only needs two booster doses, thus eliminating the need for RIG and saving money, as RIG is a relatively expensive biological. Another means of reducing cost in rabies prophylaxis is intradermal (ID) vaccination. By using small vaccine doses of $0.1 \mathrm{~mL}$ in countries where ID administration is registered, a vial of vaccine can be used for several patients. In a variety of Asian countries, including Thailand, India, Sri Lanka, and the Philippines, ID PEP is commonly used. It has been shown to be safe, immunogenic and efficacious $[4,5]$. Cost saving can be significant, when a sufficient number of patients is treated with one vial of vaccine within up to 6 hours after reconstitution. This of course is important for people in developing countries with low income, who have difficulties affording expensive PEP. ID administration of vaccine, however, is not limited to PEP. Also PrEP can be administered via the ID route [6].

Rabies PrEP is commonly used for people at occupational risk. Such risk for instance exists for veterinarians and veterinary students, bat and wildlife handlers, hunters, and laboratory personnel handling live rabies virus. In addition, a significant number of travelers from developed countries visiting high endemic areas get vaccinated before starting their journey. PrEP has been shown to be immunogenic and safe. Failures in reaching adequate antibody concentrations to date have practically not been seen in healthy individuals. The only documented cases of PrEP failure are seen in immunodepressed persons. While travelers to rabies endemic countries often get vaccinated before starting their journey, the risk of being exposed is even higher for people living in these areas. Currently, PrEP is not used large scale in any country, whether in adults or as part of pediatric vaccination campaigns, e.g. as part of national immunization programs. As RIG might not be available everywhere in developing countries, or might not be affordable, PrEP can be a means of reducing need for RIG, which consequently reduces the cost of PEP. In addition, even in circumstances where PrEP costs might be slightly higher in costs for the society or the individual, it can save lives, as not every animal bite patient gets proper PEP without delay.

When comparing PrEP with other means of rabies prevention, it is obvious that PrEP alone will not solve the root of the problem, the circulation of rabies in dogs (or otherreservoir species). But dog vaccination or elimination campaigns have not been proven to be successful either. Extensive culling, as performed in China in 2009, has not reduced the burden of disease, and is widely objected. Animal vaccination and sterilization programs also have only partly been successful and are expensive. In contrast to that, intradermal PrEP as part of a large scale vaccination program could be affordable and significantly reduce the burden of disease in humans in areas where incidences are high. The best approach in our opinion is a holistic approach, which includes dog vaccination, increase of human education and awareness, responsible dog ownership and mandatory pet dog vaccination, as well as proper prophylaxis, given before or after an exposure has occurred.

\section{References}

1. Knobel DL, Cleaveland S, Coleman PG, Fèvre EM, Meltzer MI, et al. (2005) Re-evaluating the burden of rabies in Africa and Asia. Bull World Health Organ 83: $360-368$

2. WHO (2012) Rabies. Fact sheet.

3. Wilde $\mathrm{H}$, Chutivongse $\mathrm{S}$, Tepsumethanon W, Choomkasien P, Polsuwan C, et al. (1991) Rabies in Thailand: 1990. Rev Infect Dis 13: 644-652.

4. Briggs DJ, Banzhoff A, Nicolay U, Sirikwin S, Dumavibhat B, et al. (2000) Antibody response of patients after postexposure rabies vaccination with small intradermal doses of purified chick embryo cell vaccine or purified Vero cell rabies vaccine. Bull World Health Organ 78: 693-698.

5. Quiambao BP, Dimaano EM, Ambas C, Davis R, Banzhoff A, et al. (2005) Reducing the cost of post-exposure rabies prophylaxis: efficacy of $0.1 \mathrm{ml}$ PCEC rabies vaccine administered intradermally using the Thai Red Cross post-exposure regimen in patients severely exposed to laboratory-confirmed rabid animals. Vaccine 23: 1709-1714.

6. WHO (2010) Rabies vaccines: WHO position paper. Wkly Epidemiol Rec 85 309-320.

*Corresponding author: Claudius Malerczyk, MD. Novartis Vaccines and Diagnostics GmbH, Emil-von-Behring-Str. 76, 35041 Marburg, Germany, Tel: (+49) 6421 - 39 6453; Fax: (+49)6421 - 39 6081; E-mail: claudius@malerczyk@novartis

Received November 15, 2012; Accepted November 16, 2012; Published November 20, 2012

Citation: Malerczyk C (2012) Rabies Pre-Exposure Vaccination in Rabies Endemic Countries. J Vaccines Vaccin 3:e114. doi:10.4172/2157-7560.1000e114

Copyright: (c) 2012 Malerczyk C. This is an open-access article distributed under the terms of the Creative Commons Attribution License, which permits unrestricted use, distribution, and reproduction in any medium, provided the original author and source are credited. 